\title{
Potencia térmica en un quemador experimental utilizando cáscaras de nuez Pecan como biocombustible
}

Ing. Rodolfo Oscar Berberi ${ }^{(1)}$, Ing. Sergio Smoisman ${ }^{(2)}$, Denis Sandoval ${ }^{(3)}$, Nicolás Rey ${ }^{(4)}$, Mauricio Arenas ${ }^{(4)}$, Nicolás Killy ${ }^{(4)}$, Lucas Federico Piatti ${ }^{(4)}$

\section{Resumen}

Son presentados en este documento los resultados de la investigación de la potencia térmica de un quemador experimental utilizando como combustible cáscaras de nuez Pecán, residuo que la agroindustria produce.

Palabras clave: Quemador, Pecán, potencia térmica.

\section{Introducción}

La energía térmica procede mayoritariamente de la quema de hidrocarburos y por tratarse de energía no renovable se pone en valor la consideración de combustibles alternativos, como ser las cáscaras de nuez Pecán. Su utilización contribuye a la disminución de la dependencia de los combustibles fósiles, reduciendo la contaminación ambiental y los costos de producción energética.

El objetivo de esta investigación ha sido evaluar la cantidad de energía calórica desarrollada por un quemador de biomasa experimental, diseñado y materializado por alumnos de la Universidad de la Marina Mercante.

El quemador está constituido por un sistema de alimentación, una cámara de combustión, donde se produce la incineración del combustible, un equipo de inyección de aire y un tablero de control. [Ref. 1]

E1 sistema de alimentación (Figura 1), está conformado por una tolva vertical que facilita la caída por gravedad del combustible hacia el tornillo alimentador de $10 \mathrm{~mm}$ de paso que gira dentro de un tubo transportador de $50 \mathrm{~mm}$ de diámetro, dosificando la cantidad de combustible requerida por el quemador.

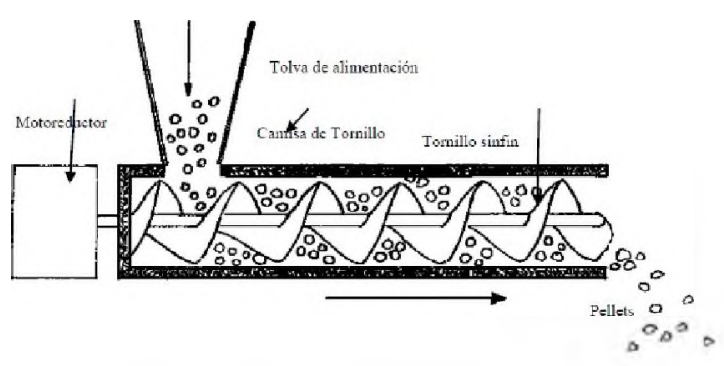

Figura 1 - Quemador de biomasa sólida

${ }^{(1)}$ Director del Grupo de Investigación, (2)Docente UTN - FRBA / UdeMM, ${ }^{(3)}$ Alumno UTN - FRBA, ${ }^{(4)}$ Alumnos UdeMM - Facultad de Ingeniería 
La velocidad de giro del tornillo, varía entre 15 y $40 \mathrm{rpm}$ y se ajusta al caudal de alimentación necesario para obtener la mejor mezcla de aire - combustible.

El encendido o ignición inicial del combustible se logra mediante una resistencia eléctrica capaz de brindar temperatura suficientemente elevada para que en conjunto con el aire generen la combustión. El combustible se encenderá solo al alcanzar su temperatura mínima de ignición, generando en una primera instancia brasas, las cuales mediante transferencia por contacto se irá propagando al resto del combustible y en conjunto con el aire inyectado por el ventilador, se obtendrá el encendido de la biomasa.

La resistencia hueca de cerámica del quemador, permite el paso del flujo de aire a temperatura ambiente $y$ en todo su trayecto es calentado hasta llegar al extremo opuesto a una temperatura entre $900^{\circ} \mathrm{C}$ y $1000^{\circ} \mathrm{C}$ (ver Figura 2).

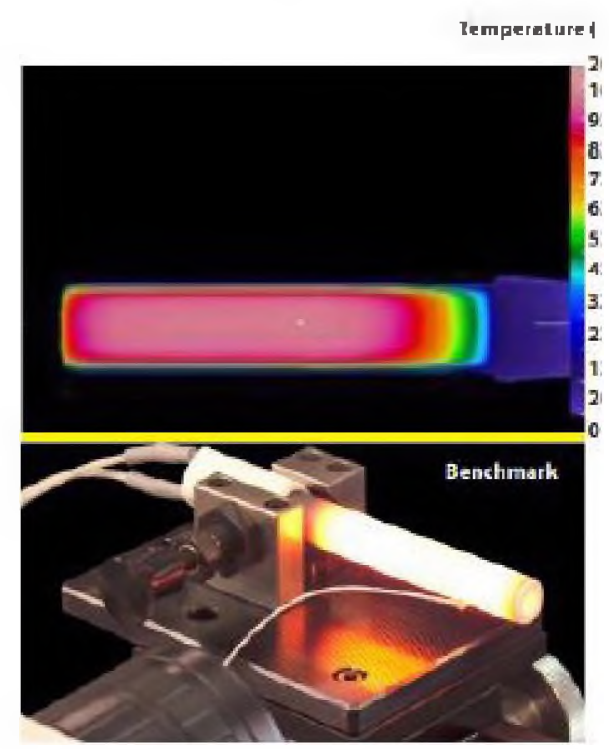

Figura 2 - Resistencia cerámica
El sistema de inyección de aire aporta un caudal de $0,0054 \mathrm{~m} 3 / \mathrm{min}$ a $0,0113 \mathrm{~m} 3 /$ $\min$.

Desde el panel de control es accionada la resistencia eléctrica y dos elementos de regulación: uno de ellos, destinado al control del caudal de combustible y el otro, al ajuste del flujo de aire para la combustión.

En la Figura 3 se observa el quemador experimental objeto de la investigación.

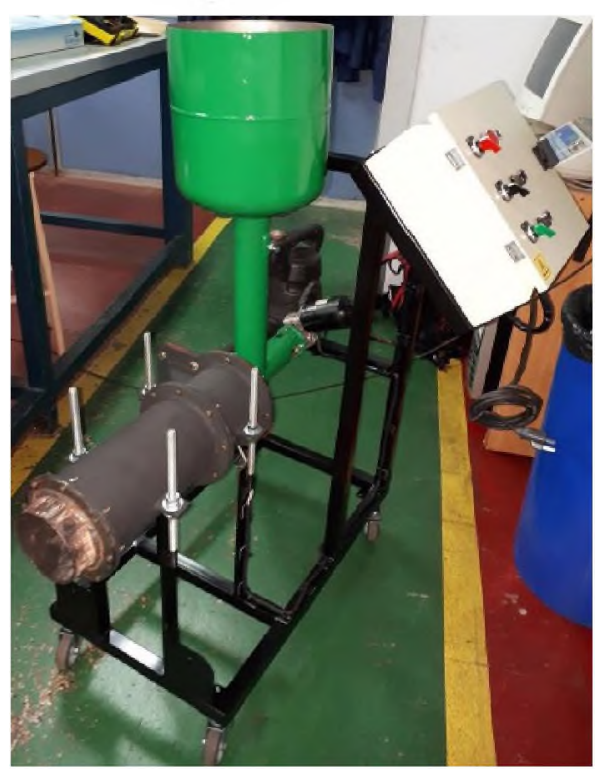

Figura 3 - Quemador de biomasa

Las cáscaras de nuez Pecán utilizadas en la investigación corresponden a la variedad MAHAN, se caracterizan por tener $52 \mathrm{~mm}$ de longitud promedio y un rinde del $41 \%$.

\section{Metodología}

Para el desarrollo de este estudio ha sido utilizado el método heurístico basado en la experiencia, la práctica y la observación de los hechos, con el fin de llegar a una solución eficaz de un problema determinado. 
Como disciplina científica puede ser aplicada a cualquier ciencia con el propósito de concluir en un resultado eficiente al problema planteado.

\section{Ensayos}

\section{"Condiciones de ensayo"}

Antes de comenzar los ensayos, se midieron la temperatura y la humedad relativa del ambiente con la asistencia de un higrómetro digital (Figura 4).

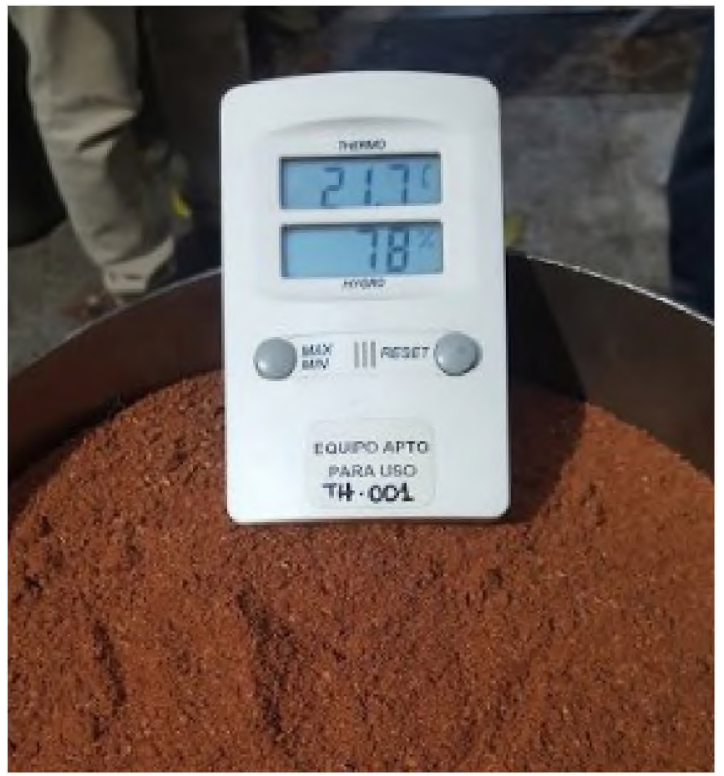

Figura 4 - Higrómetro

Los valores obtenidos fueron registrados en la Tabla 1.

Tabla 1: Condiciones ambientales

\begin{tabular}{|c|c|}
\hline Ta $\left({ }^{\circ} \mathrm{C}\right)$ & $\underline{\text { Hr }}(\%)$ \\
\hline 21,7 & 78,0 \\
\hline
\end{tabular}

"Respuesta del quemador a las cáscaras sin procesar"

Se procedió al encendido del quemador alimentándolo con $2 \mathrm{~kg}$ de cáscaras. Se ob- servó que solo una mínima parte de las mismas logró alcanzar la cámara de combustión, quedando atascadas en el tubo vertical ubicado debajo de la tolva (ver Figura 5).

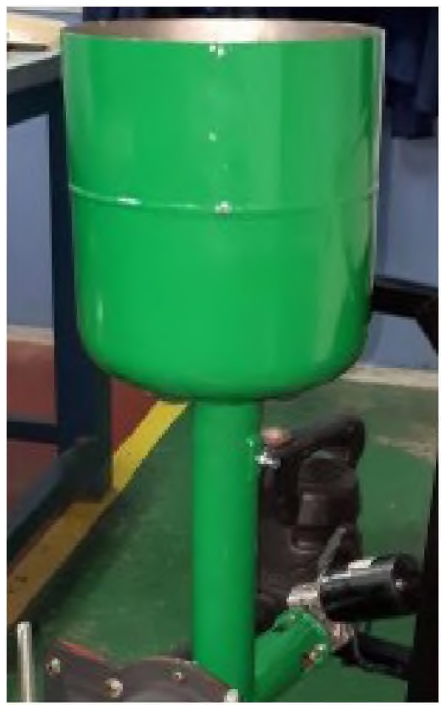

Figura 5 - Tolva y tubo vertical

A1 ser el tamaño de las cáscaras de nuez inadecuadas para la operación del quemador, debieron ser trituradas y tamizadas de acuerdo al método tamices de ensayo [Ref. 2].

Las cáscaras inicialmente sin triturar (Figura 6), fueron procesadas a fin de reducir su tamaño (Figura 7).

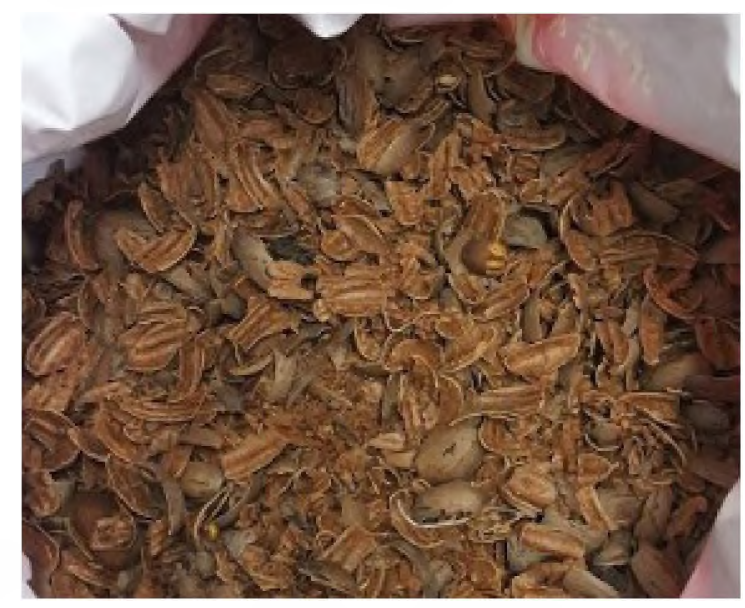

Figura 6 - Combustible triturado 


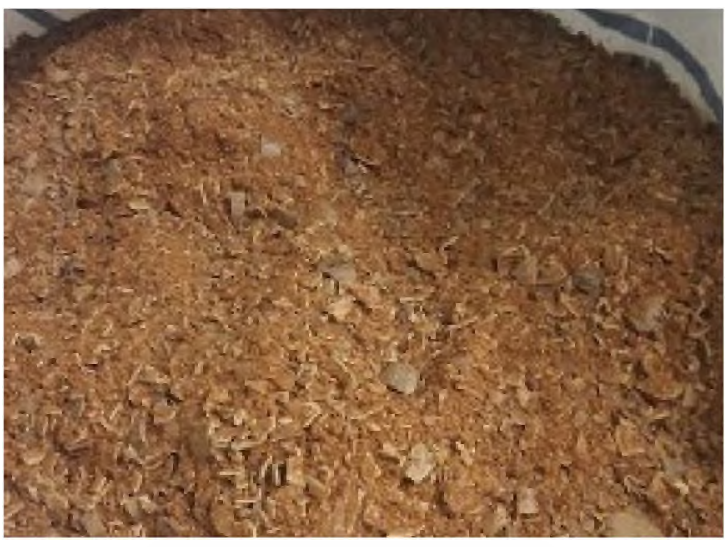

Figura 7 - Combustible molido

Luego, por intermedio de tamices normalizados, se realizó la separación obteniendo las granulometrías indicadas en la Tabla 2.

Tabla 2: Granulometrías

\begin{tabular}{|c|c|}
\hline Tamiz & Tamaño de partícula (mm) \\
\hline$\# 4$ & 4,8 \\
\hline$\# 8$ & 2,4 \\
\hline$\# 16$ & 1,2 \\
\hline
\end{tabular}

"Flujo másico útil $\left(\mathrm{G}_{\mathrm{u}}\right)$ )"

Para cada granulometría se introdujo en la tolva una masa (M) de combustible conocida. La quema resultó incompleta, ya que se presentó material inquemado (mi) Figura 8. En la Tabla 3 se muestran los porcentajes de combustible sin quemar.

Tabla 3: Porcentaje de inquemados

\begin{tabular}{|l|c|c|c|c|}
\hline $\begin{array}{l}\text { 量 } \\
\text { 恶 }\end{array}$ & $\begin{array}{c}\mathrm{M} \\
(\mathrm{kg})\end{array}$ & $\begin{array}{c}\mathrm{mi} \\
(\mathrm{kg})\end{array}$ & $\mathrm{m}_{\mathrm{u}}=\mathrm{M}$-mi & $\begin{array}{c}\% \sin \\
\text { quemar }\end{array}$ \\
\hline$\# 4$ & $\mathbf{1 , 0 0}$ & 0,30 & 0,70 & 30 \\
\hline$\# 8$ & 1,00 & 0,27 & 0,73 & 28 \\
\hline$\# 16$ & 1,00 & 0,20 & 0,80 & 20 \\
\hline
\end{tabular}

Experimentalmente se determinó cual fue la cantidad de cáscaras por unidad de tiempo $\left(\mathrm{G}_{\mathrm{T}}\right)$ que el tornillo alimentador suministró al quemador.

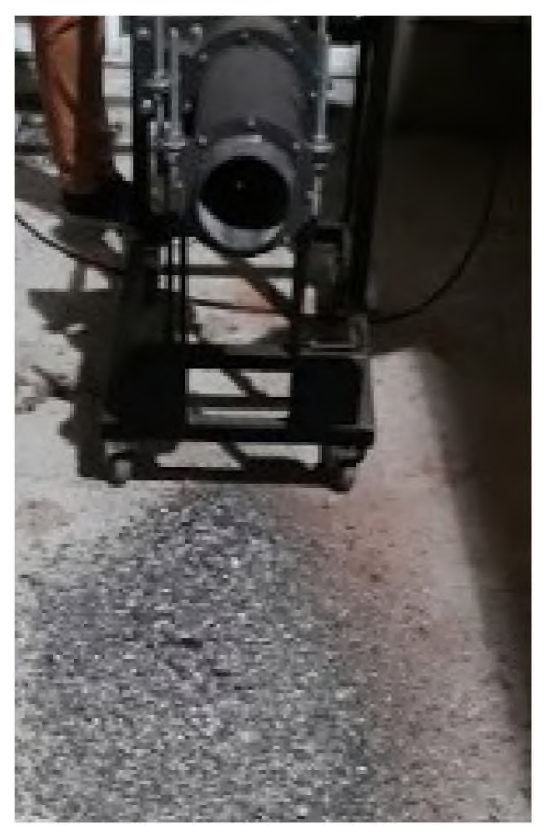

Figura 8 - Cenizas e inquemados

Con los porcentajes obtenidos de cáscaras inquemadas $\left(G_{i}\right)$, se calcula el flujo másico útil de combustible $\left(\mathrm{G}_{\mathrm{u}}\right)$ que ha sido quemado, ver Tabla 4.

Tablal 4: Flujo másico útil

\begin{tabular}{|c|c|c|c|}
\hline \multirow{2}{*}{$\begin{array}{c}\mathrm{G}_{\mathrm{T}} \\
(\mathrm{kg} / \mathrm{h})\end{array}$} & $\begin{array}{c}\dot{\mathrm{G}}_{\mathrm{l}} \\
(\%)\end{array}$ & $\begin{array}{c}\mathbf{G}_{\mathrm{u}} \\
(\mathrm{kg} / \mathrm{h})\end{array}$ \\
\hline$\# 4$ & 15,60 & 0,70 & 10,92 \\
\hline$\# 8$ & 29,29 & 0,72 & 21,09 \\
\hline$\# 16$ & $\mathbf{5 0 , 9 6}$ & $\mathbf{0 , 8 0}$ & $\mathbf{4 0 , 7 7}$ \\
\hline
\end{tabular}

"Determinación de la Potencia Calorífica del quemador (Q)"

Para verificar el potencial como combustible fue consultado el análisis elemental de la cáscara Pecán elaborado por 
Antal y colaboradores (Tabla 5), quienes utilizaron un equipo de Análisis Elemental CHONS marca Huffman y una metodología dictada por la norma ASTM D1762-84 [Ref. 3].

Tabla 5: Composición de la Nuez Pecán. Fuente Antal y cols. (2000)

\begin{tabular}{|c|c|}
\hline Elemento & Porcentaje (\%) \\
\hline $\mathrm{C}$ & $\mathbf{5 5 , 2 7}$ \\
\hline $\mathrm{H}$ & $\mathbf{4 , 5 6}$ \\
\hline $\mathrm{N}$ & $\mathbf{0 , 8 4}$ \\
\hline $\mathrm{S}$ & $\mathbf{0 , 0 9}$ \\
\hline $\mathrm{O}$ & $\mathbf{3 4 , 7 5}$ \\
\hline Cenizas & $\mathbf{5 , 8 5}$ \\
\hline Carbón Fijo & $\mathbf{3 4 , 9}$ \\
\hline
\end{tabular}

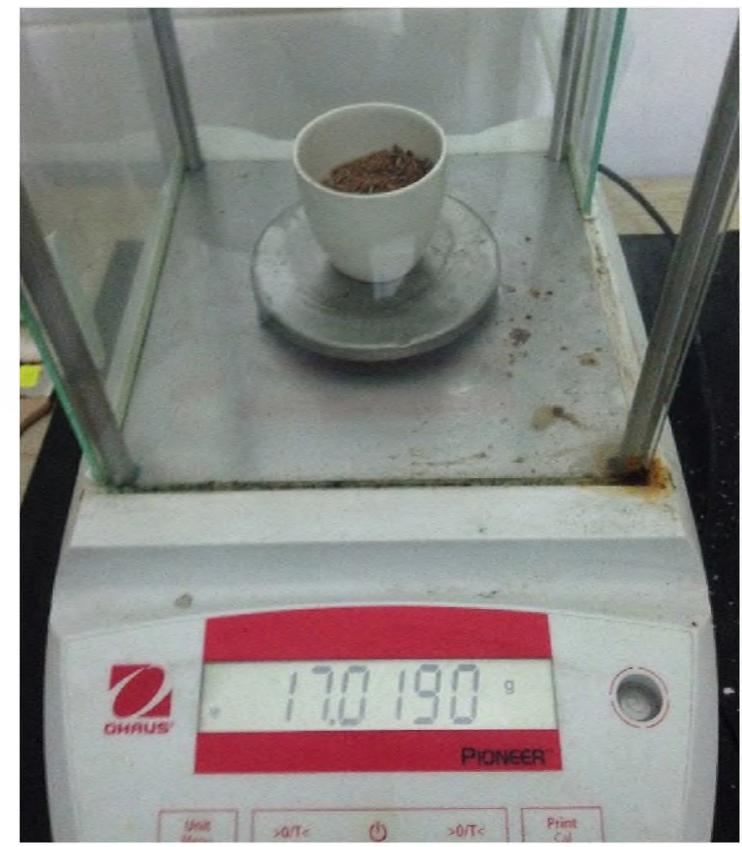

Figura 9-Muestra analizada

Tabla 6: Medición de humedad de las cáscaras de nuez ensayadas

\begin{tabular}{|c|c|c|c|}
\hline Medición & $\begin{array}{l}\text { Peso de } \\
\text { cascara } \\
\text { [gr.] }\end{array}$ & $\begin{array}{l}\text { Contenido } \\
\text { de agua } \\
\text { [gr.] }\end{array}$ & $\begin{array}{l}\text { Polcentaje } \\
\text { de } \\
\text { humedad } \\
{[\%]}\end{array}$ \\
\hline $\begin{array}{l}\text { Estado } \\
\text { inicial }\end{array}$ & 17,019 & 0 & 0 \\
\hline 1 & 16,048 & 0,9701 & 5,70011 \\
\hline 2 & 15,329 & 1,6893 & 9,92596 \\
\hline 3 & 14,924 & 2,0947 & 12,3080 \\
\hline 4 & 14,437 & 2,5817 & 15,1695 \\
\hline 5 & 14,383 & 2,6353 & 15,4844 \\
\hline 6 & 14,334 & 2,6847 & 15,7747 \\
\hline
\end{tabular}

Las cáscaras ensayadas presentaron un contenido de humedad aproximado de $15,8 \%$.

Para los combustibles sólidos se tiene la fórmula de Dulong:

$P c i=8100 . C+29000\left(H-\frac{O}{8}\right)+2500 . S-600 W$ 


\section{$P c i=3880 \mathrm{Kcal} / \mathrm{kg}$}

Con los valores obtenidos del poder calorífico inferior y el flujo másico útil, fue calculada la potencia térmica del quemador (Q) para cada granulometría utilizada (ver Tabla 7).

Tabla 7: Potencia térmica

\begin{tabular}{|c|c|c|c|}
\hline \multicolumn{4}{|c|}{$\dot{Q}=P_{c i} * \dot{G}_{u}$} \\
\hline $\begin{array}{c}\text { Tami } \\
\mathbf{z}\end{array}$ & $\begin{array}{c}\text { Pci } \\
\text { (kcal/kg } \\
\text { ) }\end{array}$ & $\begin{array}{c}G_{u} \\
(\mathrm{~kg} / \mathrm{h} \\
)\end{array}$ & $\begin{array}{c}\dot{Q} \\
(\mathrm{kcal} / \mathrm{h})\end{array}$ \\
\hline$\# 4$ & \multirow{3}{*}{3880} & 10,92 & $42.396,60$ \\
\hline$\# 8$ & & 21,09 & $81,829,20$ \\
\hline \#16 & & 40,77 & $\begin{array}{c}158.187,6 \\
0\end{array}$ \\
\hline
\end{tabular}

\section{Conclusiones}

- Cuanto menor es la granulometría del combustible mayor es la potencia térmica entregada por el quemador, debido a que las cáscaras se quemaron a mayor velocidad.

- El diseño del quemador no favoreció la producción de brasas en la etapa de ignición con granulometrías menores a

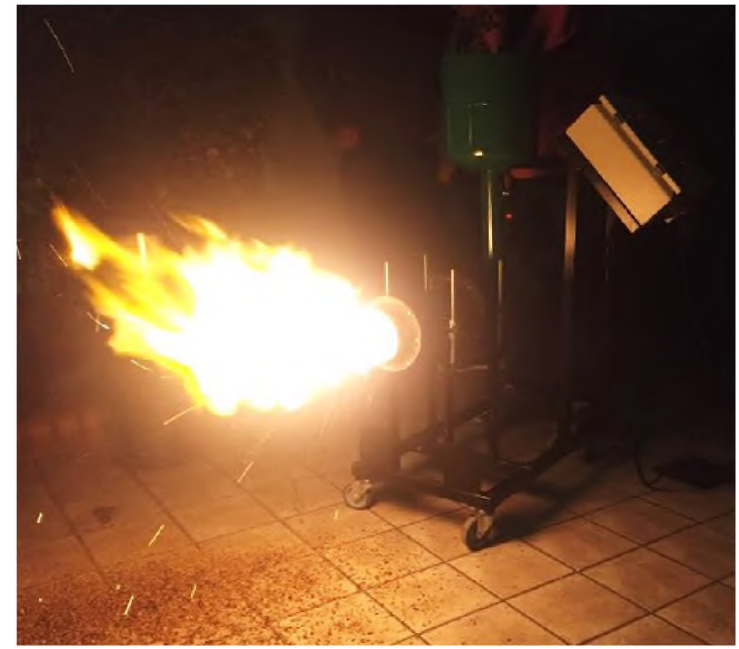

Figura 10 - quemador experimental

1,2mm (Tamiz \#16); al mismo tiempo, debió regularse el caudal de aire para reducir la cantidad de combustible inquemado.

- Para las condiciones ambientales de ensayo y granulometrías igual o menor a 1,2 mm (Tamiz \#16), se observó el fenómeno de aglomeración impidiendo la circulación continua desde la tolva hacia el tornillo alimentador.

- A partir del poder calorífico inferior obtenido, el bajo porcentaje de inquemados y la potencia calórica calculada, se concluye que las cáscaras de nuez Pecan tienen el potencial para ser consideradas como combustible alternativo en el quemador experimental ensayado. 


\section{Referencias}

[1] Proyecto Final Universidad de la Marina Mercante (2017). "Proyecto de Integrado Quemador de Biomasa".

[2] Instituto Argentino de Normalización y Certificación (IRAM). N 1501 "Tamices de ensayo".

[3] Publicación en la Universidad de Vallado- lid (2016). "Aprovechamiento de Residuos Agroforestales, con particular interés en los originados en explotaciones de Nuez Pecán (Carya illinoinensis), por medio del cultivo de Hongos Saprófitos Saludables Ibéricos". España.

[4] American Society for Testing and Materials (ASTM). N C566-97 "Métodos de medición de humedad". 